\title{
Masquerading Mycobacterium: Plastic surgeon to the rescue
}

\author{
V Kumar MB BS MRCS (Glasgow), MSE Coady FRCS(Plastic surgery), R Taranu MB ChB
}

V Kumar, MSE Coady, $\mathrm{R}$ Taranu. Masquerading Mycobacterium: Plastic surgeon to the rescue. Can J Plast Surg 2005;13(1)36-38.

A patient with a Mycobacterium marinum infection of the hand is described. The present case illustrates that $\mathrm{M}$ marinum infection may mimic common skin conditions such as eczema, and fungal and parasitic infestations. Key elements in the diagnosis and management of this infection are a high index of suspicion, a detailed history of recreational or occupational exposure to exotic fish, tissue biopsy, wound culture and prompt empirical antibiotic therapy. Once in vitro organism sensitivities are obtained, antibiotic treatment may last for up to 24 months. Surgical drainage and debridement are an important supplement to antimicrobial therapy when subcutaneous structures are involved, as in the present report. Invasive digital and hand infections can rapidly result in permanent residual stiffness, deformity and loss of function; therefore, it is important to raise awareness of the condition among those considered to be at risk.

Key Words: Fish tank granuloma; Immunosuppresion; Mycobacterium

\section{CASE PRESENTATION}

A 41-year-old woman presented to her general practitioner in March 2001 with a painless thickening of the skin over the dorsal aspect of the proximal interphalangeal joint (PIPJ) of her right dominant middle finger (Figure 1). Initial referral to secondary care provided a diagnosis of eczematous reaction and the patient was referred to a dermatologist. Topical therapy failed, and after a few unsuccessful courses of antibiotics, clinical suspicion of a granulomatous condition arose. On direct questioning, the patient revealed the existence of an exotic fish aquarium in her house which she had cleaned on a number of occasions. No specific injury to the skin was recognized. A presumptive diagnosis of 'fish tank granuloma' was made and the patient was then referred to the plastic surgery hand service.

In October 2002, while awaiting her outpatient appointment, she presented acutely with subcutaneous fluctuance over the right middle finger PIPJ. On presentation, the patient had a temperature of $35.8^{\circ} \mathrm{C}$. Prompt incision and drainage revealed a subcutaneous, supratendinous dorso-ulnar abscess with mustard-coloured pus. There were signs of chronic

\section{Mycobacterium démasqué : Plasticien à la rescousse}

On décrit ici le cas d'un patient atteint d'une infection à Mycobacterium marinum au niveau de la main. Ce cas illustre que l'infection à $M$. marinum peut prendre l'apparence de maladies dermatologiques, comme l'eczéma, ou d'infestations fongiques et parasitaires. Les éléments clés du diagnostic et du traitement de cette infection sont tout d'abord un fort indice de soupçon, une anamnèse détaillée des activités récréatives et professionnelles ayant pu mener à l'exposition à des poissons exotiques, la biopsie tissulaire, la culture de plaie et l'instauration rapide d'une antibiothérapie empirique. Une fois les résultats des cultures obtenus, l'antibiothérapie peut durer jusqu'à 24 mois. Le drainage et le débridement chirurgicaux sont d'importants compléments à l'antibiothérapie lorsque les structures sous-cutanées sont touchées, comme dans le cas présent. L'infection invasive des doigts et de la main peut rapidement entraîner une raideur, une déformation et une perte de fonction résiduelles permanentes. Par conséquent, il est important de sensibiliser la communauté médicale à cette maladie chez les sujets à risque.

Department of Plastic Reconstructive Surgery, The James Cook University Hospital, Middlesbrough, United Kingdom Correspondence: Mr Vinod Kumar, Department of Orthopaedics, The James Cook University Hospital, Middlesbrough, United Kingdom

TS4 3BW. Telephone 0164-285-0850 or 0779-314-0651, fax 0164-285-4375, e-mail geo_bug@yahoo.com 


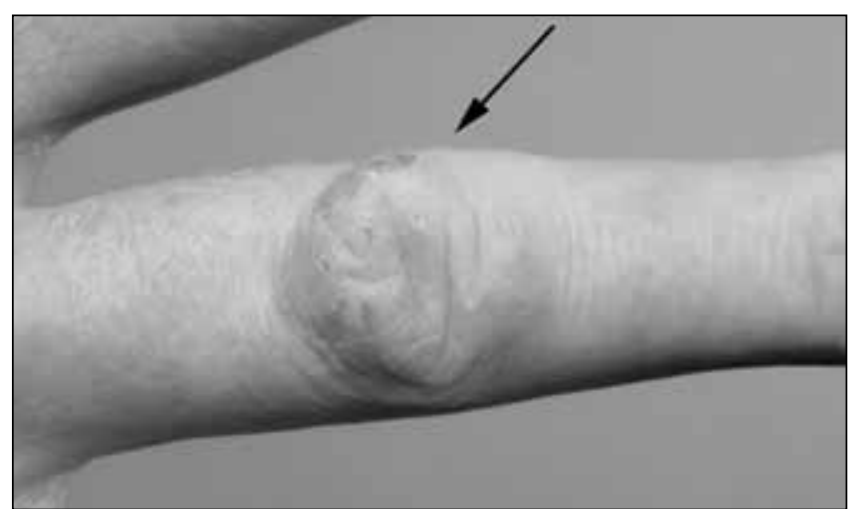

Figure 1) Erythematous plaque over the proximal interphalangeal joint of the middle finger

mycobacteria are a heterogenous group of acid-fast bacilli which were not recognized as potential human pathogens until 35 to 40 years ago. In general, they form an important part of bacterial flora in our environment. As a group, they differ in several respects from the classic tubercle bacilli; they present with varying degrees of acid fastness, have a wider temperature range for growth, growth rates are more variable, human infections are frequently associated with pre-existing disease or trauma, and they show a general pattern of resistance to the first-line antituberculous drugs such as ethambutol, streptomycin and isoniazid. The Runyon classification of nontuberculous mycobacteria is based on the rate of growth, production of yellow pigment and whether this pigment was produced in the dark or only after exposure to light. On this basis, they are divided into four groups (Table 1).

$\mathrm{M}$ marinum has a worldwide distribution. There are various vectors of human infection including salt and fresh water fish, dolphins, shrimps, oysters and snails (1). M marinum infection occurs following major or minor skin trauma, usually to an extremity, which is then either immersed in contaminated water or comes into direct contact with marine creatures (2). Numerous cases of infection have been reported as being acquired from contaminated pools, rivers and old wells (1), and from sources as diverse as dolphin bites and laboratory cultures (3). Exposure to $\mathrm{M}$ marinum in swimming pools is rare because most are chlorinated (2). The organism grows best at $30^{\circ} \mathrm{C}$ to $32^{\circ} \mathrm{C}$, which partially explains why cooler extremities are more often affected than central structures.

$M$ marinum infections may be an inadvertent occupational hazard for certain professions such as pet shop workers. Most infections are acquired during the handling of aquariums when cleaning or changing the water (1), although infection may result from direct injury (fish fins or bites) (4). Nosocomial infection has never been described (2). The international incidence and prevalence rates are unknown due to the lack of surveillance. It belongs to the same group of nontuberculous mycobacteria as Mycobacterium kansasii, Mycobacterium aviumintracellulare and Mycobacterium fortuitum complex, infections caused by which are usually self-limiting, but are responsible for progressive and, if untreated, usually fatal disease in immunocompromised individuals.

Clinically, a bluish nodule or papule develops at the inoculation site. Patients may also present with an erythematous plaque, particularly on their hands, as in the case described. Ulceration may occur later, and subsequent lesions may be
TABLE 1

Runyon classification of nontuberculous Mycobacterium species

\begin{tabular}{lll}
\hline Group 1 & Photochromogenic & M marinum \\
Group 2 & slow growers & M kansasii \\
& scotochromogenic & $M$ scrofulaceum \\
Group 3 & Nonphotochromogenic & M gordonae \\
& slow growers & $M$ avium-intracellulare \\
Group 4 & Rapid growers & $M$ xenopi \\
\end{tabular}

present along the path of lymphatic drainage of the extremity (sporotrichoid spread) (5). Patients may experience more invasive infection; tenosynovitis (6), septic arthritis and osteomyelitis have been reported. It rarely disseminates except in the severely immunocompromised. An upper extremity is affected in nearly $90 \%$ of cases. Most patients respond to a tuberculin skin test with less than $10 \mathrm{~mm}$ induration.

Diagnosis is frequently delayed because of the rarity of the condition and failure to elicit a history of exposure. Common differential diagnoses with which the condition can be confused are fungal and parasitic infections, gout, rheumatoid arthritis, and foreign body and cutaneous malignancies. Tissue biopsy and culture to isolate the organism are crucial to confirm the diagnosis. While the presence of a cutaneous granuloma alerts the clinician to the possibility of mycobacterial infection, they are not specific to $M$ marinum. Histopathologically, early lesions show epidermal hyperkeratosis, a mixed inflammatory response, or possibly frank suppuration; however, caseation is not encountered (2).

The microbiologist must be alerted to the suspicion of $\mathrm{M}$ marinum so that the specimens are cultured in the appropriate manner. Most strains of $\mathrm{M}$ marinum grow best at $30^{\circ} \mathrm{C}$ to $33^{\circ} \mathrm{C}$ using the Loweinstein-Jensen medium. They do not grow at $37^{\circ} \mathrm{C}$, the usual incubation temperature for Mycobacterium tuberculosis. After seven to 14 days of culture, microscopy reveals nonmotile acid-fast bacilli (1).

Spontaneous resolution has been reported (2), but treatment is usually with bactericidal agents, supplemented by appropriate surgical procedures for deeper infections. The organism is resistant to the traditional antituberculous medications isoniazid, streptomycin and para-aminosalicylic acid (2). It is, at present, usually sensitive to rifampicin, ethambutol, tetracyclines, clarithromycin, levofloxacin (7), ciprofloxacin (8) and trimethoprim-sulphamethoxazole (2). Clarithromycin can be used for initial therapy before obtaining microbiological sensitivities, as can ethambutol and rifampicin combined. The duration of therapy is empirical, but should continue for four to six weeks following clinical resolution of lesions. Treatment may be up to 18 months or longer for deeper infections.

Although uncommonly encountered, skin lesions caused by $\mathrm{M}$ marinum are not rare. However, in its initial stages this condition does mimic other commonly encountered cutaneous diseases or cutaneous manifestations of systemic diseases. An awareness of the potential for cutaneous mycobacterial infection will prompt relevant history taking, early diagnosis and treatment. Promptly commencing appropriate antimicrobial therapy combined with tissue biopsy and culture for more 
definitive diagnosis will avert significant patient morbidity. People working near salt water and aquariums or home aquarium owners, particularly if immunosuppressed, should be instructed to follow simple measures such as wearing gloves when cleaning out tanks and cleansing skin thoroughly with antibacterial soaps if bites or abrasions occur. Persistent, unexplained red or bluish thickening of the skin in at-risk individuals should arouse suspicion and be investigated further. With the increasing number of immunocompromised patients we encounter in clinical practice, it is likely that we will see a rising trend in these infections. A high index of suspicion is the key to the diagnosis.

\section{REFERENCES}

1. Bhatty MA, Turner DP, Chamberlain ST. Mycobacterium marinum hand infection: Case reports and review of literature. Br J Plast Surg 2000;53:161-5.
2. Raphael JK. Mycobacterium marinum. http://www.emedicine.com/med/topic1538.htm. (Version current at December 2, 2004)

3. Kullavanijaya P, Sirimachan S, Bhuddhavudhikrai P. Mycobacterium marinum cutaneous infection acquired from occupations and hobbies. Int J Dermatol 1993;32:504-7.

4. Hurst LC, Amadio PC, Badalamente MA, Ellstein JL, Dattwyler RJ. Mycobacterium marinum infections of the hand. J Hand Surg [Am] 1987;12:428-35.

5. Feddersen A, Kunkel J, Jonas D, Engel V, Bhakdi S, Husmann M. Infection of the upper extremity by Mycobacterium marinum in a 3-year-old boy - diagnosis by $16 \mathrm{~S}$-rDNA analysis. Infection 1996;24:47-8.

6. Lim YW, Chia J, Looi KP. A case report of Mycobacterium marinum infection of the hand. Singapore Med J 2000;41:221-3.

7. Iijima S, Saito J, Otsuka F. Mycobacterium marinum skin infection successfully treated with levofloxacin. Arch Dermatol 1997;133:947-9.

8. Laing RB, Flegg PJ, Watt B, Leen CL. Antimicrobial treatment of fish tank granuloma. J Hand Surg [Br] 1997;22:135-7. 